1

\title{
Symbiosis increases population size and buffers environmental fluctuations in a physiologically-structured model parameterized for thyasirid bivalves
}

\author{
Joany Mariño ${ }^{\mathrm{a},}$, Suzanne C. Dufour ${ }^{\mathrm{a}}$, Amy Hurford ${ }^{\mathrm{a}, \mathrm{b}}$ \\ ${ }^{a}$ Department of Biology, Memorial University of Newfoundland. St. John's A1B 3X9, \\ Canada \\ ${ }^{b}$ Department of Mathematics and Statistics, Memorial University of Newfoundland. St. \\ John's A1C 5S7, Canada
}

\begin{abstract}
Symbioses whereby one partner provisions a nutritional resource to the other may alter energy allocation towards reproduction and survival in the recipient partner, potentially impacting population dynamics. Asymbiotic thyasirid bivalves feed predominantly on free-living bacteria, which fluctuate in abundance due to seasonality-driven temperature variations. Symbiotic thyasirids are mixotrophs, gaining nutrients from free-living bacteria and symbiotic bacteria that they host on their enlarged gills. Symbiotic bacteria may function as an additional energy reserve for thyasirids, allowing the hosts to allocate more energy to reproduction. We hypothesize that, for symbiotic thyasirids, the symbionts are a nutritional source that mitigates resource limitation. Using Dynamic Energy Budget theory, we built a physiologically-structured population model assuming equal mortality rates in both species. We find that without seasonal fluctuations, symbiotic thyasirids have higher abundances than asymbiotic thyasirids since the symbionts increase reproduction. Both species have similar population sizes in fluctuating environments, suggesting different adaptations to seasonality: asymbiotic thyasirids have adapted their physiology, while symbiotic thyasirids have adapted through mixotrophy. Our results highlight the significance of linking individual energetics and life-history traits to population dynamics and are the first step towards understanding the role of symbioses in population and community dynamics.
\end{abstract}

Email address: jmarinocoron@mun.ca (Joany Mariño) Preprint submitted to bioRxiv 
Keywords: Energy reserves, dynamic energy budget theory, mixotrophy, thyasirids, seasonality, structured population model, symbiosis

\section{Introduction}

Nutritional symbiosis is a prevalent interaction that can increase the metabolic capabilities of the host (Dubilier et al., 2008; Moran, 2006). Hence, symbiosis has the potential to affect host life-history traits, such as fecundity and survival, which, in turn, determine population dynamics. However, how symbiosis can influence host ecology and how this would be translated into population and community dynamics is not known (Yule et al., 2013). Disentangling the bottom-up effect of trophic symbiosis on ecological timescales, in both constant and heterogeneous environments, is crucial to understanding the conditions that lead to the persistence of populations and communities (Miller and Rudgers, 2014).

Environmental heterogeneity and the pattern of environmental variation are thought to be determinant factors in the evolution of the niche breadth, particularly for traits such as foraging strategies (Lynch and Gabriel, 1987; Kassen, 2002). Theory suggests that selection favours generalist strategies in populations experiencing environmental heterogeneity (Lynch and Gabriel, 1987; Levins, 1968; Futuyma and Moreno, 1988). Experimental results have confirmed such findings (Reboud and Bell, 1997; Bell and Reboud, 1997; Kassen and Bell, 1998, but see Riddle et al. 1986 for conflicting results). For example, selection experiments in Chlamydomonas in constant environments have led to the evolution of specialists, either autotrophic or heterotrophic. Conversely, in temporally varying environments, selection favours generalists capable of both autotrophic and heterotrophic nutrition. However, in spatially varying environments, both specialists can be retained in the population (Reboud and Bell, 1997; Bell and Reboud, 1997; Kassen and Bell, 1998). Broadly, these results suggest that ecological specialists tend to be selected in environments that are homogeneous in space or time, whereas generalists tend to favoured in temporally varying environments (Kassen, 2002: Lynch and Gabriel. 1987; Levins, 1968; Futuyma and Moreno, 1988; Ackermann and Doebeli, 2004).

Nutritional symbioses in which the host has a mixotrophic nutrition (i.e. the host can combine the nutritional input from the symbionts with heterotrophic or autotrophic feeding; Rossi et al., 2017) can be considered to 
be generalist feeding strategies. For instance, mixotrophic symbioses are frequent in marine suspension feeders, which live in environments where light and plankton concentration are variable and often limiting (Muller-Parker and Davy, 2001; Grottoli et al., 2006). In octocorals, such trophic flexibility has been proposed to maximize nutrient uptake, allowing for increased energy acquisition, relative to asymbiotic species (Gori et al., 2012; Grottoli et al., 2006). Moreover, the loss of symbionts may not significantly affect the host's energetic input. making the host less affected by environmental variability (Rossi et al., 2017: Viladrich et al., 2017; Fabricius et al., 1995; Sorokin, 1991; Ferrier-Pagès et al., 2015). Thus, in seasonal environments, symbionts can provide energy to a mixotrophic host and stabilize the discontinuous energy inputs in resource availability (Rossi et al., 2017; Viladrich et al., 2017; Gori et al., 2012).

Another notable example of mixotrophy occurs in symbiotic thyasirid bivalves, a family that stands out for including symbiotic as well as asymbiotic members (Taylor et al., 2007; Southward, 1986). Symbiotic thyasirids are flexible mixotrophs that can digest their symbionts depending on environmental conditions, particularly the presence of sulfide and external particulate food (Dando and Spiro, 1993; Dufour and Felbeck, 2006). Evidence in other bivalves suggests that changes in the relative importance of different food sources are likely correlated to particulate food abundance (Pile and Young, 1999). A mixotrophic nutrition is considered a strategy that allows symbiotic thyasirids to thrive in fluctuating environments (Dufour and Felbeck, 2006; Duperron et al., 2013).

In the fjord of Bonne Bay (Newfoundland, Canada), two species of thyasirids are sympatric and have a patchy distribution. The first species resembles Thyasira gouldi (in shell characteristics and internal anatomy) and therefore is referred to as T. cf. gouldi; the second species, Parathyasira sp., is asymbiotic. Both symbiotic T. cf. gouldi and asymbiotic Parathyasira are particulate feeders that rely on chemoautotrophic bacteria as their primary resource (60\% and $70 \%$, respectively), with lesser contributions of suspended and particulate organic matter (Zanzerl et al., 2019). However, rather than collecting chemoautotrophic bacteria from sediments through pedal feeding, T. cf. gouldi harbours these bacteria extracellularly as symbionts on enlarged gills and digests them as an additional resource (Laurich et al., 2015; Zanzerl et al., 2019); hence, it is considered a mixotrophic species. For T. sarsi, another mixotrophic thyasirid, between 26 and $76 \%$ of their nutrition has been estimated to be obtained from the bacterial symbionts (Dando and Spiro, 
1993). Given that the carbon isotope composition in T. cf. gouldi overlaps the lower range of the isotopic signature of T. sarsi (Dando and Spiro, 1993; Zanzerl et al., 2019), it is likely that the reliance of $T$. cf. gouldi on symbionts may be similar to the lower limit of T. sarsi's, comprising approximately $25 \%$ of their diet. For thyasirids inhabiting an environment with strong seasonality, temperature and resource fluctuations will affect the individual metabolic rates and the costs associated with maintaining the symbionts. Previous theoretical research showed that symbiotic T. cf. gouldi has a smaller energy reserve, which implies reduced energy assimilation and mobilization fluxes, lower somatic maintenance costs and growth rate, and more significant energy allocation to maturity and reproduction (Mariño et al., 2019). However, how the nutritional differences between symbiotic T. cf. gouldi and asymbiotic Parathyasira are reflected at the population and community levels is not known.

Our previous results support the hypothesis that a mixotrophic (generalist) strategy results in higher energy allocation to reproduction (McKillup and Butler, 1979; Thompson, 1983; George, 1994; Viladrich et al., 2017). Thus, in a constant environment, a mixotrophic population should have larger abundances than the asymbiotic (specialist) population. Here, we hypothesize that when there is seasonality, relying on symbionts will buffer the fluctuations in resource availability for the host population. We predict that if symbionts effectively mitigate resource variability, then the mixotrophic population will be less prone to extinction during winter when the abundance of free-living bacteria becomes limiting. Our prediction should hold while the abundance of symbionts is not zero and is at least equal to the lowest free-living bacterial abundance. However, the buffering effect of the symbionts should decrease as the host becomes more specialized and increases reliance on the symbionts. Hence, in highly seasonal environments, a low or intermediate level of dependence on symbionts (i.e. a generalist strategy) should be favoured over the specialist strategy.

Since the physiological responses of individuals can be considered the underlying basis of their ecological dynamics, models that consider the organismal bioenergetics are powerful tools to understand how energetic constraints determine changes in the niche of a species as a consequence of environment fluctuations (Nisbet et al., 2012). Energy budget models describe the rates at which an individual assimilates and uses energy for maintenance, growth, and reproduction (van der Meer, 2006; Kooijman, 2010). To understand how the differences due to feeding and symbiosis translate to the population level 
and shape host population dynamics, we combine the individual-level energy budget dynamics with a physiologically-structured population model (Kooijman, 2010; De Roos et al., 2009). We built a population model that accounts for the species' physiology according to the individual DEB model and takes into account the seasonal pattern of temperature and resource abundance. Using this model, we simulated the dynamics of the symbiotic T. cf. gouldi and the asymbiotic Parathyasira.

Previously, we suggested that the energy allocation patterns in symbiotic thyasirids may represent an evolutionary strategy where the symbionts function as a partial energy reserve, allowing the individuals to invest more energy in reproduction (Mariño et al., 2019). Here, we focus on the differences in populations inhabiting a constant versus seasonal environment, and evaluate different scenarios of symbiont dependence and abundance. We show how the symbiotic strategy is likely to mitigate the effects of environmental variability in a population of symbiotic thyasirids. We discuss the buffering effect of the symbionts in terms of the evolution and ecological adaptation of thyasirids and mixotrophic bivalves.

\section{Methods}

\subsection{The model}

We formulated a continuous population model that focuses on the representation of individual physiology and life history. To describe individuals, we used the DEB-abj model (Kooijman, 2014), which is structured by energy, volume, maturity and reproduction (Fig. 1). We used published data from T. cf. gouldi and Parathyasira sp. to parameterize the model (Mariño et al., 2019). We assumed that individuals in the population could exploit one or two resources, depending on whether they are asymbiotic or symbiotic. To explicitly include the dependency of the resource on the environmental temperature, we modelled the resource according to relationships derived from the Metabolic Theory of Ecology (Savage et al., 2004). To test our predictions of how symbiosis affects populations of thyasirid bivalves, we conducted numerical simulations for T. cf. gouldi and Parathyasira in different environmental conditions that consider various temperature and resource availability scenarios. To further analyze the possible effects of symbiosis on populations of $T$. cf. gouldi, we carried out simulations representing different relative symbiont abundances and different contribution of symbionts to the host's diet. 


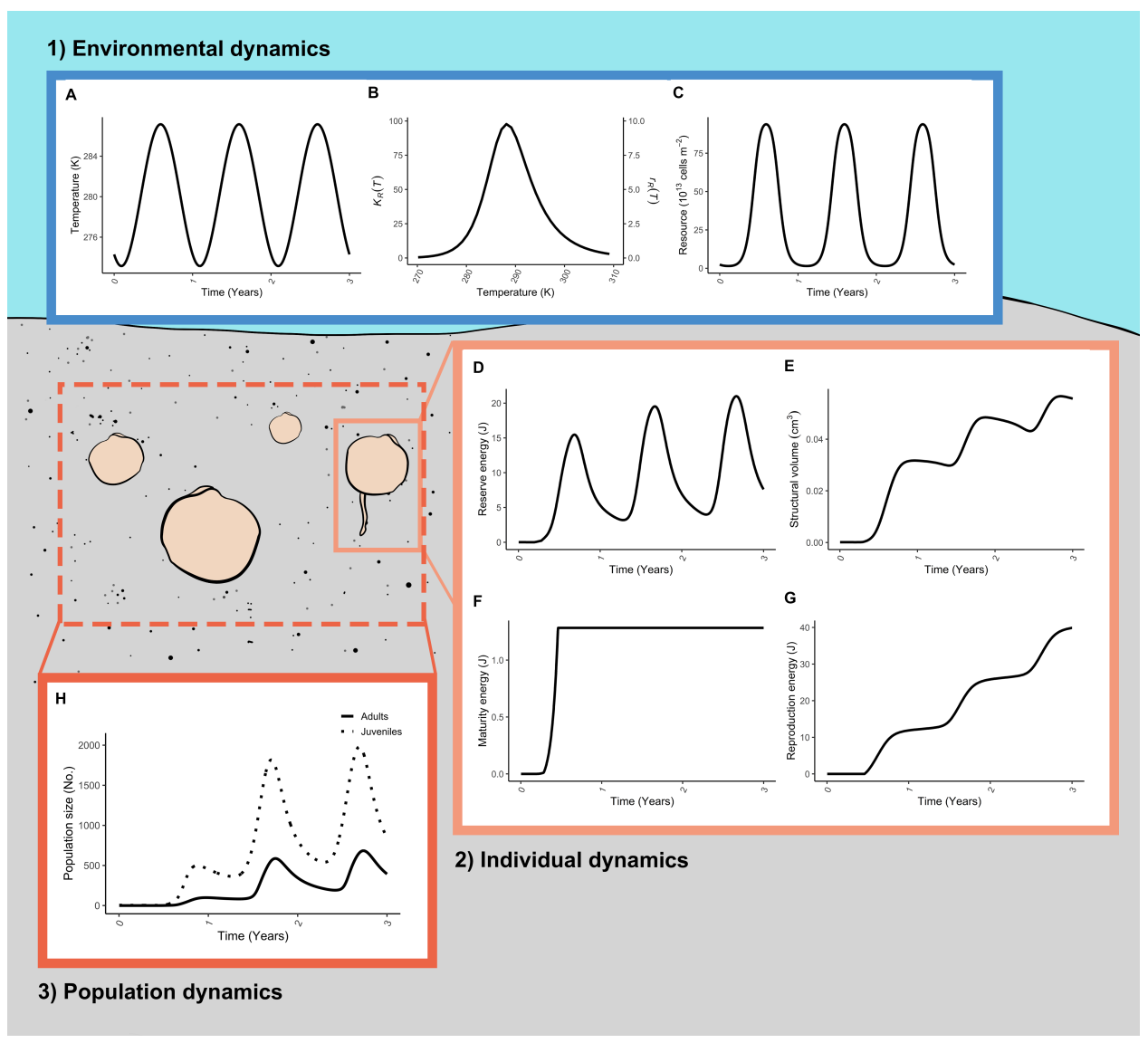

Figure 1. Representation of the three main components of the model for Thyasira cf. gouldi in time: 1) the environment (A, B, C), 2) the individual (D, E, F G) and 3) the population $(\mathrm{H})$. The environmental variables of temperature (A, equation 1$)$ and free-living bacterial resource $(\mathrm{C}$, equation 2) determine each individual's dynamics. The carrying capacity of the free-living bacterial resource $\left(K_{R}(T)\right)$ and its growth rate $\left(r_{R}(T)\right)$ are functions of the environmental temperature (B, equations 3 and 4 ). For both parameters, we follow relationships derived from the Metabolic Theory of Ecology (Savage et al., 2004). We characterize the individuals by four variables: energy in reserve (D), structural volume (E), maturity (F) and reproduction energy $(\mathrm{G})$, according to the DEB model (equation 7). We obtain the population dynamics $(\mathrm{H})$ by numerically integrating over all the individuals (equation 10). The simulations' parameters are given in Tables 2 and 3. We assumed an initial condition of 5 embryos for the simulation of the population. 


\subsection{Environment}

\subsubsection{Temperature}

We modelled an annual cycle that corresponds to the seafloor temperatures at Bonne Bay, which range from 0.7 to $14{ }^{\circ} \mathrm{C}$, approximately (Laurich et al., 2015, see Fig. 1 A). More specifically, the temperature at time $t$ oscillates around the average temperature $(\bar{T})$ with amplitude $T_{a}$ and period equal to the length of the year:

$$
T(t)=\bar{T}+T_{a} \sin \left(2 \pi \frac{t}{365}\right) .
$$

For comparative purposes, we also modelled a constant environment assuming that the temperature is equal to the mean annual temperature, $\bar{T}$.

\subsubsection{Resource}

The primary resource for T. cf. qouldi and Parathyasira is free-living, chemoautotrophic bacteria (Zanzerl et al., 2019). In cold, marine sediments, such as Bonne Bay, bacterial production is typically seasonal, with specific growth rates increasing with temperature; further, bacterial production is directly proportional to bacterial biomass (Sander and Kalff, 1993). Thus, we assumed that the free-living bacterial resource $R$ is a function of the environmental temperature $T$, and follows logistic growth:

$$
R(T)=r_{R}(T) R\left(\frac{K_{R}(T)-R}{K_{R}(T)}\right),
$$

where $r_{R}$ is the resource growth rate and $K_{R}$ is the maximum resource density. For notational simplicity, we let $T=T(t)$; however, it should be understood that temperature may be a function of time as described in equation 1 .

To include the dependence of the resource on the environmental temperature, we described both resource parameters using relationships derived from the Metabolic Theory of Ecology (Savage et al., 2004). We assumed that the growth rate and carrying capacity increase exponentially with temperature. Further, around the limits of the bacteria's thermal niche, both parameters drop steeply to zero, according to a Sharpe-Schoolfield term (Schoolfield 
et al., 1981, see Fig. 1B). Hence, the resource growth rate is given by:

$$
\begin{aligned}
r_{R}(T)= & r_{0} e^{\left(-\mathcal{E} / k_{B}\right)\left(1 / T-1 / T_{0}\right)}\left(1+e^{\left(-\mathcal{E}_{L} / k_{B}\right)\left(1 / T-1 / T_{L}\right)}\right. \\
& \left.+e^{\left(-\mathcal{E}_{H} / k_{B}\right)\left(-1 / T+1 / T_{H}\right)}\right)^{-1}
\end{aligned}
$$

where $r_{0}$ is the resource growth rate at a reference temperature $T_{0}, k_{B}$ is the Boltzmann constant, $\mathcal{E}$ is the average activation energy driving resource growth at intermediate temperatures, $\mathcal{E}_{L}$ and $\mathcal{E}_{H}$ are the inactivation energies that determine the slope of the resource growth rate as it drops to zero at the lower and upper thermal tolerance limits, $T_{L}$ and $T_{H}$, respectively.

The maximum resource density follows a similar formulation:

$$
\begin{aligned}
K_{R}(T)= & K_{0} e^{\left(-\mathcal{E} / k_{B}\right)\left(1 / T-1 / T_{0}\right)}\left(1+e^{\left(-\mathcal{E}_{L} / k_{B}\right)\left(1 / T-1 / T_{L}\right)}\right. \\
& \left.+e^{\left(-\mathcal{E}_{H} / k_{B}\right)\left(-1 / T+1 / T_{H}\right)}\right)^{-1}
\end{aligned}
$$

where $K_{0}$ is the maximum resource density at a reference temperature $T_{0}$. As with the resource growth rate, the parameters $\mathcal{E}, \mathcal{E}_{L}$ and $\mathcal{E}_{H}$ represent the temperature sensitivity of the maximum resource density within and outside of the lower and upper temperature thresholds, $T_{L}$ and $T_{H}$.

\subsection{Individual dynamics}

We described the individual life history and physiology (i.e. feeding, growth, and reproduction) as a function of the individual state variables and the state of the environment.

\subsubsection{Feeding}

All individuals forage on the resource (free-living bacteria) following a functional response $f(t, T, R)$, which is a function of the time of the year, the temperature and the resource abundance. Asymbiotic individuals feed only on one resource according to the scaled functional response:

$$
f_{A}(R)=\frac{R(T)}{R_{\max }},
$$

where $R_{\max }$ is the maximum resource density. 
Symbiotic individuals can forage on the symbionts as an additional resource, which we modelled as a constant, scaled abundance $S$. Thus, the functional response for symbiotic individuals $f_{S}(t, T, R)$ includes their reliance on symbionts $\alpha$, and is given by:

$$
f_{S}(R)=(1-\alpha)\left(\frac{R(T)}{R_{\max }}\right)+\alpha\left(\frac{S}{R_{\max }}\right) .
$$

\subsubsection{Growth and reproduction: DEB-abj model}

The individual dynamics are according to the Dynamic Energy Budget theory (Kooijman, 2010). This approach distinguishes between the biomass of the organism that functions as an energy reserve and as structure. Specifically, we use the DEB-abj model, which is a one parameter extension of the standard DEB model that accounts for a growth pattern recognized in bivalves termed metabolic acceleration (Kooijman, 2014).

Each individual is characterized by four state variables: energy in reserve $(E)$, structural volume $\left(L^{3}\right)$, cumulative energy invested into maturation $\left(E_{H}\right)$, and cumulative energy invested into reproduction $\left(E_{R}\right)$. The dynamic of the individual in time is given by:

$$
\begin{gathered}
\frac{d E}{d t}=\dot{p}_{A}-\dot{p}_{C}, \\
\frac{d L^{3}}{d t}=\kappa\left(\dot{p}_{C}-\dot{p}_{S}\right) /\left[E_{G}\right], \\
\begin{cases}\frac{d E_{H}}{d t}=(1-\kappa) \dot{p}_{C}-\dot{p}_{J} \text { and } \frac{d E_{R}}{d t}=0, & \text { if }\left(E_{H}<E_{H}^{p}\right), \\
\frac{d E_{H}}{d t}=0 \text { and } \frac{d E_{R}}{d t}=(1-\kappa) \dot{p}_{C}-\dot{p}_{J}, & \text { otherwise, }\end{cases}
\end{gathered}
$$

where the energy fluxes are denoted by $\dot{p}$ (see Table 1), $\kappa$ is the fraction of mobilized reserve allocated to somatic metabolism, $\left[E_{G}\right]$ is the specific cost to grow one unit of structure, and $E_{H}^{p}$ is the puberty threshold. 
Table 1. Energy fluxes $(\dot{p}, \mathrm{~J} / \mathrm{d})$ and shape correction function $(\mathcal{M})$ at each developmental stage. Each stage is defined according to the cumulative maturity thresholds $E_{H}^{b}, E_{H}^{j}$ and $E_{H}^{p}$, which represent birth, metamorphosis, and puberty, respectively. The scaled functional response is $f\left(0 \leq f \leq 1\right.$, where 1 is the highest amount of food), $\left\{\dot{p}_{\mathrm{Xm}}\right\}$ is the maximum surface-area specific ingestion rate $\left(\left\{\dot{p}_{\mathrm{Xm}}\right\}=\left\{\dot{p}_{\mathrm{Am}}\right\} / \kappa_{X}, \mathrm{~J} /\right.$ day $\cdot$ $\mathrm{cm}^{2}$, for a maximum surface-area specific assimilation rate $\left.\left\{\dot{p}_{\mathrm{Am}}\right\}\right), \kappa_{X}$ is the assimilation efficiency from food to reserve, $\dot{v}$ is the energy conductance rate from the energy reserve (cm/day), $\dot{r}$ is the individual growth rate (1/day, equation 8$),\left[\dot{p}_{M}\right]$ is the volume-specific somatic maintenance cost $\left(\mathrm{J} /\right.$ day $\left.\cdot \mathrm{cm}^{3}\right), \dot{k}_{J}$ is the maturity maintenance rate coefficient. The structural lengths at the beginning and at the end of the acceleration are $L_{b}$ and $L_{j}$, which correspond to the structural lengths at birth and at metamorphosis. Notation: square brackets ([ ]) indicate quantities related to structural volume, curly brackets $(\{\})$ denote quantities related to structural surface-area, dots $\left(^{\circ}\right)$ indicate rates.

\begin{tabular}{lcccc}
\hline Flux & $\begin{array}{c}\text { Embryo } \\
\left(E_{H} \leq E_{H}^{b}\right)\end{array}$ & $\begin{array}{c}\text { Early juvenile } \\
\left(E_{H}^{b}<E_{H} \leq E_{H}^{j}\right)\end{array}$ & $\begin{array}{c}\text { Late juvenile } \\
\left(E_{H}^{j}<E_{H}<E_{H}^{p}\right)\end{array}$ & $\begin{array}{c}\text { Adult } \\
\left(E_{H} \geq E_{H}^{p}\right)\end{array}$ \\
\hline Feeding, $\dot{p}_{X}$ & 0 & $f\left\{\dot{p}_{\mathrm{Xm}}\right\} \mathcal{M}$ & $f\left\{\dot{p}_{\mathrm{Xm}}\right\} \mathcal{M}$ & $f\left\{\dot{p}_{\mathrm{Xm}}\right\} \mathcal{M}$ \\
Assimilation, $\dot{p}_{A}$ & $\kappa_{X} \dot{p}_{X}$ & $\kappa_{X} \dot{p}_{X}$ & $\kappa_{X} \dot{p}_{X}$ & $\kappa_{X} \dot{p}_{X}$ \\
Mobilization, $\dot{p}_{C}$ & $E \dot{v}(\mathcal{M} / L-\dot{r})$ & $E \dot{v}(\mathcal{M} / L-\dot{r})$ & $E \dot{v}(\mathcal{M} / L-\dot{r})$ & $E \dot{v}(\mathcal{M} / L-\dot{r})$ \\
Soma maint., $\dot{p}_{S}$ & {$\left[\dot{p}_{M}\right] L^{3}$} & {$\left[\dot{p}_{M}\right] L^{3}$} & {$\left[\dot{p}_{M}\right] L^{3}$} & {$\left[\dot{p}_{M}\right] L^{3}$} \\
Maturity maint., $\dot{p}_{J}$ & $\dot{k}_{J} E_{H}$ & $\dot{k}_{J} E_{H}$ & $\dot{k}_{J} E_{H}$ & $\dot{k}_{J} E_{H}^{p}$ \\
Shape function, $\mathcal{M}$ & $L_{b} / L_{b}=1$ & $L / L_{b}$ & $L_{j} / L_{b}$ & $L_{j} / L_{b}$ \\
\hline
\end{tabular}

The growth rate for each individual is given by:

$$
\dot{r}=\frac{\kappa \frac{E}{L^{4}} \dot{v} \mathcal{M}-\left[\dot{p}_{M}\right]}{\kappa \frac{E}{L^{3}}+\left[E_{G}\right]},
$$

where $\mathcal{M}$ is a shape correction function that varies according to the stage of the individual (see Table 1), $\dot{v}$ is the energy conductance rate, and $\left[\dot{p}_{M}\right]$ is the somatic maintenance cost.

The energy fluxes for all the metabolic rates are temperature-dependent (see Section 1.3 in Kooijman, 2010), therefore the parameters of the model are standardized to a reference temperature of $20^{\circ} \mathrm{C}$. The correction between the reference temperature and the empirical temperature $T$ is done through the Arrhenius relationship:

$$
c_{T}=\exp \left(\frac{T_{A}}{T_{\text {ref }}}-\frac{T_{A}}{T}\right)
$$

where $c(T)$ is the correction factor for a certain temperature $T, T_{A}$ is the 
Arrhenius temperature and $T_{r e f}$ is the reference temperature. For example, the mobilization flux (Table 1 ) at temperature $T$ becomes: $\dot{p}_{C}(T)=\dot{p}_{C}\left(T_{r e f}\right)$. $c(T)$.

Table 2. Parameter values for the individual-level dynamics (equation 7) for the symbiotic Thyasira cf. gouldi and the asymbiotic Parathyasira sp. (Mariño et al., 2019). Notation: square brackets ([ ]) indicate quantities related to structural volume, curly brackets $(\{\})$ denote quantities related to structural surface-area, dots $\left(^{\cdot}\right)$ indicate rates.

\begin{tabular}{lcccc}
\hline Description & Symbol & Thyasira cf. gouldi & Parathyasira & Unit \\
\hline Maximum assimilation rate & $\left\{\dot{p}_{\mathrm{Am}}\right\}$ & 1.427 & 2.547 & $\mathrm{~J} / \mathrm{day} \cdot \mathrm{cm}^{2}$ \\
Assimilation efficiency & $\kappa_{X}$ & 0.8 & 0.8 & - \\
Energy conductance rate & $\dot{v}$ & 0.02 & 0.02 & $\mathrm{~cm} / \mathrm{day}$ \\
Allocation fraction to soma & $\kappa$ & 0.883 & 0.958 & - \\
Somatic maintenance cost & {$\left[\dot{p}_{M}\right]$} & 15.78 & 23.61 & $\mathrm{~J} / \mathrm{day} \cdot \mathrm{cm}^{3}$ \\
Maturity maintenance coefficient & $\dot{k}_{J}$ & 0.002 & 0.002 & $1 / \mathrm{day}^{3}$ \\
Specific cost for structure & {$\left[E_{G}\right]$} & 2355 & 2348 & $\mathrm{~J} / \mathrm{cm}^{3}$ \\
Maturity at birth & $E_{H}^{b}$ & $2.639 \mathrm{e}-4$ & $7.193 \mathrm{e}-5$ & $\mathrm{~J}$ \\
Maturity at metamorphosis & $E_{H}^{j}$ & 0.011 & 0.002 & $\mathrm{~J}$ \\
Maturity at puberty & $E_{H}^{p}$ & 1.283 & 0.96 & $\mathrm{~J}$ \\
Weibull aging acceleration & $\dot{h}_{a}$ & $9.844 \mathrm{e}-8$ & $1.262 \mathrm{e}-7$ & $1 / \mathrm{day}^{2}$ \\
Shape coefficient & $\delta_{M}$ & 0.507 & 0.64 & - \\
Arrhenius temperature & $T_{A}$ & 8000 & 8000 & $\mathrm{~K}$ \\
Reference temperature & $T_{\mathrm{ref}}$ & 293.15 & 293.15 & $\mathrm{~K}$ \\
\hline
\end{tabular}

\subsection{Population dynamics}

We represent the population by a density $n$, which is a function of the four individual DEB model (or i-states) variables $\left(n\left(t, E(t), V(t), E_{H}(t), E_{R}(t)\right)\right)$ and its dynamic in time is given by:

$$
\frac{\partial n}{\partial t}+\frac{\partial n}{\partial E} \frac{d E}{d t}+\frac{\partial n}{\partial V} \frac{d V}{d t}+\frac{\partial n}{\partial E_{H}} \frac{d E_{H}}{d t}+\frac{\partial n}{\partial E_{R}} \frac{d E_{R}}{d t}=-\mu\left(n+n^{2}\right)
$$

where $\mu$ is the per capita mortality rate. The set of all the possible i-states defines the population state space $\Omega \subset \mathbb{R}^{n}$. To prevent individuals from leaving the domain, we included no-flux boundary conditions for each i-state variable: $E_{\mid \partial \Omega} \equiv 0, V_{\mid \partial \Omega} \equiv 0, E_{H_{\mid \partial \Omega}} \equiv 0, E_{R_{\mid \partial \Omega}} \equiv 0$.

The population state space is divided into subsets that represent the different life stages of the individuals. For simplicity, we group the two 
juvenile stages together, and consider the domains $\Omega_{J}$, and $\Omega_{A}$, corresponding to the juvenile and adult stages. The boundary between these subsets is given by the cumulative maturity energy threshold parameter $E_{H}^{p}$. Here, we assume that once individuals are adults they cannot rejuvenate and decrease their maturity level. Hence, we further suppose the no-flux boundary:

$$
E_{H}(x)=\left\{\begin{array}{ll}
E_{H}(x) & \text { if } E_{H}(x) \geq 0, \\
0 & \text { otherwise, }
\end{array} \quad \forall x \in \partial \Omega_{J} .\right.
$$

The reproduction of the adult population gives the boundary condition at age zero:

$$
n\left(t, E_{0}, V_{0}, E_{H_{0}}, E_{R_{0}}\right)=\int_{\Omega_{A}} \beta n d E d V d E_{H} d E_{R},
$$

where we consider that the per capita fecundity rate $\beta$ is a constant. Additionally, we assume that individuals are born in the population at the origin of the domain.

\subsection{Model analysis}

We implemented our population model in the $\mathrm{R}$ language ( $\mathrm{R}$ Core Team, 2019) and solved it as an initial value problem through the methods of lines. For this, we discretized the evolution equations with finite differences and performed the time integration using the Isoda initial value problem solver from the package deSolve (Soetaert et al., 2010). Our simulations represented experimental populations that start with 5 embryos, for either species (as in Martin et al., 2012, 2013). Thus, the initial conditions for our numerical simulations were:

$$
n(t=0, x)= \begin{cases}5, & \text { if } x=\left(E_{0}, V_{0}, E_{H_{0}}, E_{R_{0}}\right), \\ 0, & \text { otherwise. }\end{cases}
$$

To investigate how symbiosis affects population dynamics, we conducted simulations at constant and fluctuating environments for both species. The environment and population parameters are given in Table 3, the parameters for the individual dynamics are given in Table 2. For these simulations, we assumed that the abundance of the symbionts was equal to the mean abundance of the free-living bacterial resource $(S=\bar{R})$. Further, we assumed that symbionts provide $25 \%$ of the diet of symbiotic individuals $(\alpha=0.25)$. 
Table 3. Parameter values for the environment and population-level dynamics for the symbiotic Thyasira cf. gouldi and asymbiotic Parathyasira sp. We assume that the parameter values are equal for both populations.

\begin{tabular}{|c|c|c|c|c|}
\hline Description & Symbol & Value & Unit & Reference \\
\hline \multicolumn{5}{|l|}{ Environment } \\
\hline Temperature & $T$ & variable & $\mathrm{K}$ & (Laurich et al. 2015 ) \\
\hline Mean temperature & $\bar{T}$ & 279.15 & $\mathrm{~K}$ & (Laurich et al. 2015) \\
\hline Temperature amplitude & $T_{a}$ & 7 & - & (Laurich et al., 2015) \\
\hline Lower temperature limit for survival & $T_{L}$ & 273.15 & $\mathrm{~K}$ & - \\
\hline Upper temperature limit for survival & $T_{H}$ & 303.15 & $\mathrm{~K}$ & - \\
\hline Reference temperature & $T_{0}$ & 288.15 & $\mathrm{~K}$ & $\left(T_{H}+T_{L}\right) / 2$ \\
\hline Mean activation energy & $\mathcal{E}$ & 0.43 & $\mathrm{~J}$ & (Savage et al., 2004) \\
\hline Lower tolerance limit inactivation energy & $\mathcal{E}_{L}$ & 1.9 & - & - \\
\hline Upper tolerance limit inactivation energy & $\mathcal{E}_{H}$ & 1.9 & - & - \\
\hline Boltzman constant & $k_{B}$ & $8.617 e^{-5}$ & $\mathrm{eV} / \mathrm{K}$ & - \\
\hline Free-living bacterial resource & $R$ & variable & - & - \\
\hline Free-living bacterial resource growth rate at $T_{0}$ & $r_{0}$ & $5 e^{6}$ & day $^{-1}$ & - \\
\hline Free-living bacterial resource density at $T_{0}$ & $K_{0}$ & $5 e^{7}$ & day $^{-1}$ & - \\
\hline Maximum free-living bacterial resource density & $R_{\max }$ & variable & - & - \\
\hline Symbiont scaled abundance & $S$ & variable & - & - \\
\hline \multicolumn{5}{|l|}{ Population } \\
\hline Birth rate & $\beta$ & 0.005 & day $^{-1}$ & - \\
\hline Death rate & $\mu$ & 0.001 & day $^{-1}$ & (De Roos and Persson, 2001) \\
\hline
\end{tabular}

To analyse the effect of the abundance of symbionts in a host population inhabiting a fluctuating environment, we investigated three possible cases: i) the abundance of the symbionts is equal to the average abundance of the resource $(S=\bar{R})$; ii) the symbionts are more abundant than the resource ( $S=R_{\max }$ ); and iii) the symbionts are less abundant than the resource $\left(S=R_{\min }\right)$. Here, we also assumed that the contribution of symbionts to a host individuals' diet is $25 \%(\alpha=0.25)$. Further, to assess how the reliance on symbionts alters the abundance of the host population, we evaluated the impact of the dependence on symbionts $(\alpha)$ for the case $S=\bar{R}$. This way, we consider several values of $\alpha$, ranging from individuals that do not rely on symbionts $(\alpha=0)$, to those that rely solely on symbionts as their resource $(\alpha=1)$. The results presented for these sections correspond to the values of the population after transient dynamics have disappeared. 


\section{Results}

\subsection{The symbiotic population has a greater proportion of adults}

In the constant environment, both the asymbiotic and the symbiotic $(\alpha=25 \%)$ species reach carrying capacity and stabilize after two years (Fig. 2A). Both populations are dominated by individuals in the juvenile classes, with all the stages following the same growth pattern (Fig. 2B, C). However, the symbiotic T. cf. gouldi population shows a faster growth rate and reaches a higher carrying capacity, relative to the asymbiotic Parathyasira (Fig. 2 A-C) since individuals of T. cf. gouldi allocate more energy to reproduction (Mariño et al., 2019). Further, in the symbiotic population, a larger proportion of individuals are in the adult stage (Fig. 2C).

In the seasonal environment, the populations of both species experience yearly cycles of low temperatures and low abundances of free-living bacteria (Fig. 1A, B), which cause a decrease in the individual growth and maturity rates as well as in the production of offspring (Fig. 1D, F). Consequently, both populations exhibit similar amplitude fluctuations with a one-year periodicity (Fig. 2D). During the periods of low free-living bacteria abundance, the symbiotic and asymbiotic thyasirid populations have similar sizes (Fig. 2D). Individuals in all the stages follow the same regular oscillations, with juveniles being the most abundant class in both populations (Fig. 2E). However, the proportion of adults is higher in the symbiotic population, reaching a greater abundance and a larger minimum size than the asymbiotic population (Fig. 2F).

\subsection{Increasing symbiont abundance increases the population size of the host}

In the seasonal environment, the dynamics of the population of symbiotic T. cf. gouldi vary according to the abundance of symbiotic bacteria (Fig. 3A). The population attains the largest size when the abundance of symbionts is greater than the abundance of the free-living bacteria (i.e. $S>R$, Fig. 3A). In this case, the mean population size and the amplitude of the yearly cycles are larger than in the other scenarios. As the abundance of the symbionts decreases (i.e. $S<R$ ), the host population exhibits cycles of smaller amplitudes and reaches a smaller mean size (Fig. 3A).

In the three scenarios of symbiont abundance that we considered, the dynamics of the different age classes of symbiotic T. cf. gouldi follow the same pattern as the population (Fig. $3 \mathrm{~B}$ ). Individuals in the juvenile stage dominate the populations and exhibit the largest amplitude in abundance when 
A

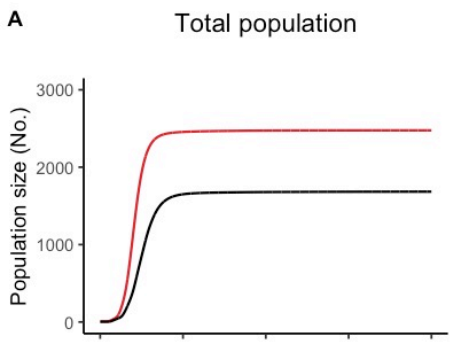

D

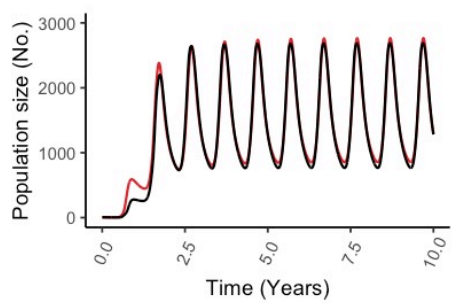

B
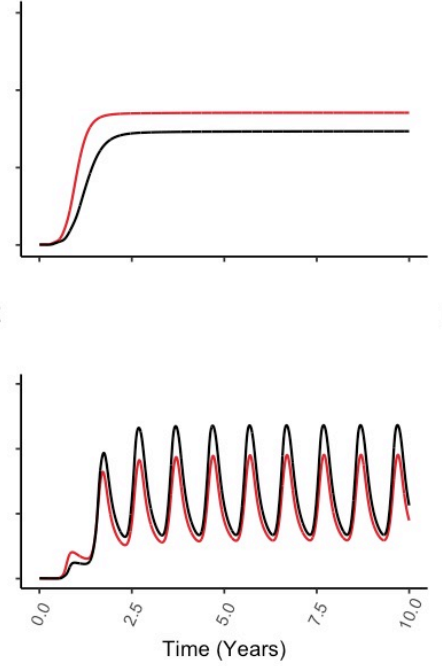

C

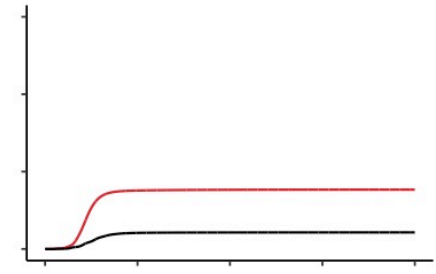

F

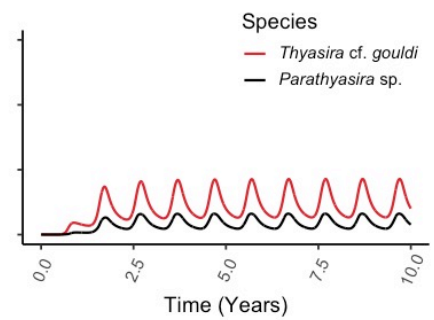

Figure 2. Modelled long term population dynamics for the symbiotic T. cf. gouldi and asymbiotic Parathyasira sp. in a constant (A-C) or fluctuating environment (D-F). For all cases, the initial population consists of five embryos. The reliance of $T$. cf. gouldi on symbionts is assumed to correspond to $25 \%$ of their diet. The symbiotic strategy allows individuals to invest more energy in reproduction, resulting in larger population sizes than the asymbiotic population in the constant environment (A). In the seasonal environment (D), both populations exhibit yearly cycles due to the combined effect of temperature and resource fluctuations. The stage dynamics for juvenile individuals (B, E) and adult individuals $(\mathrm{C}, \mathrm{F})$ exhibit the same pattern as the total population, with juveniles dominating both species' populations. In both environment scenarios, the proportion of adults is greater in the symbiotic population. 
A

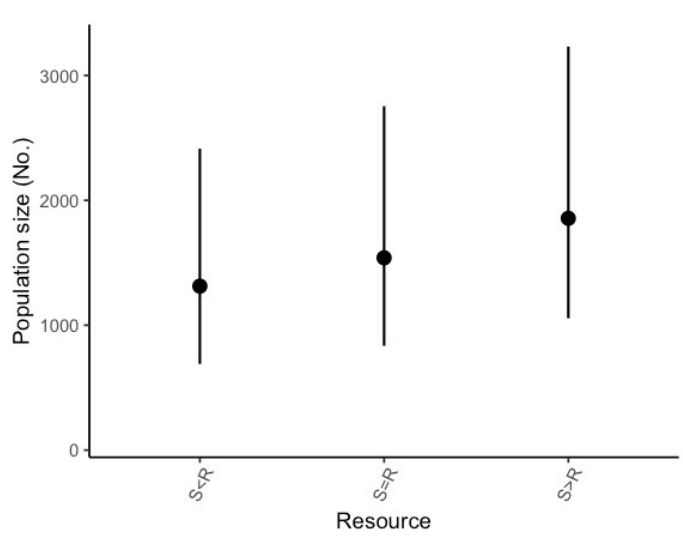

B

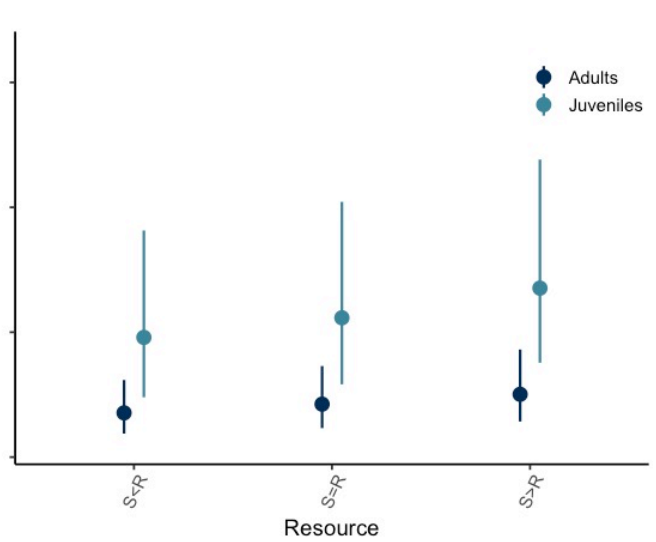

Figure 3. Effect of different symbiont abundances $(S)$ relative to the free-living bacteria $(R)$ on the host population of $T$. cf. gouldi in a seasonal environment. For all cases, the initial population consists of five embryos, and the reliance of $T$. cf. gouldi on symbionts corresponds to $25 \%$ of their diet. Points represent the average population size after discarding transient dynamics, and lines correspond to the yearly cycle's amplitude. An increasing relative symbiont abundance is predicted to increase the average, minimum and maximum population sizes in T. cf. gouldi (A). The stage dynamics exhibit the same pattern as the total population, with juveniles dominating both species' populations (B).

the conditions for the symbionts are favourable. In contrast, fluctuations in amplitude are smaller for the adult stage. Nevertheless, an increase in the abundance of symbionts favours a higher mean number of adults.

\subsection{Increasing reliance on symbionts reduces the amplitude of the population cycles of the host}

For populations of symbiotic T. cf. gouldi in a seasonal environment, the amplitude of the yearly cycles depends on the reliance of each individual host on the symbiotic bacteria (Fig. A A). The largest mean population size and annual cycles with the highest amplitude occur in the population where individuals do not obtain nutrients from symbionts (i.e. $\alpha=0$, equivalent to asymbiotic individuals, Fig. (4). An increasing reliance on symbionts reduces the effect of seasonal fluctuations in the free-living bacterial resource. Consequently, both the amplitude of the population cycles and the mean population size decrease. The smallest amplitudes and mean population sizes occur in the population where hosts rely entirely on symbionts (i.e. 
$\alpha=1$, equivalent to obligate symbiosis), given that they are not affected by fluctuations in resource availability. However, populations that rely entirely on symbionts still experience fluctuations in abundance since temperature affects the metabolic rates. Furthermore, the minimum population size rises with increasing reliance on symbionts, the highest minimum occurring in the population where individuals are obligate symbionts. Therefore, in a seasonal environment, populations with a greater dependence on symbionts are less likely to experience extinction.

As in our previous results, the dynamics of the two stages of symbiotic T. cf. gouldi follow the same fluctuating pattern as the combined population (Fig. $4 \mathrm{~B}$ ). In all the scenarios considered, juvenile individuals predominate and show the greatest variation in abundance, whereas individuals in the adult class exhibit cycles of smaller amplitude. Both the adult and juvenile stages reach a greater average population size when they have a low reliance on symbionts. Similarly, the mean population size of both stages decreases with an increase in the individual specialization on the symbionts. For the two stages, the minimum population size shows an increase as the dependence on symbionts increases.

\section{Discussion}

We show how the host's individual physiology and the abundance of and dependence on symbionts affect thyasirid population dynamics in constant and seasonal environments. Our simulations for a constant environment reveal that the mixotrophic species reaches a higher population size than the asymbiotic species. In a seasonal environment, the population of symbiotic adults has a higher growth rate during periods of the year with higher temperatures and a greater abundance of free-living resources. Similarly, in periods where the temperatures are low, and resource is limiting, the mixotrophic adult population is less prone to extinction due to the assimilation of symbiotic bacteria. Moreover, the symbiont abundance and the degree of specialization in the host's diet modulate the effect of symbiosis. Thus, our results support our initial hypothesis that symbiosis with an intermediate level of reliance on the symbionts mitigates the impact of resource seasonality.

\subsection{Population dynamics}

We found that symbiotic thyasirids reach larger population sizes and have faster population growth than the asymbiotic species in a constant environ- 
A

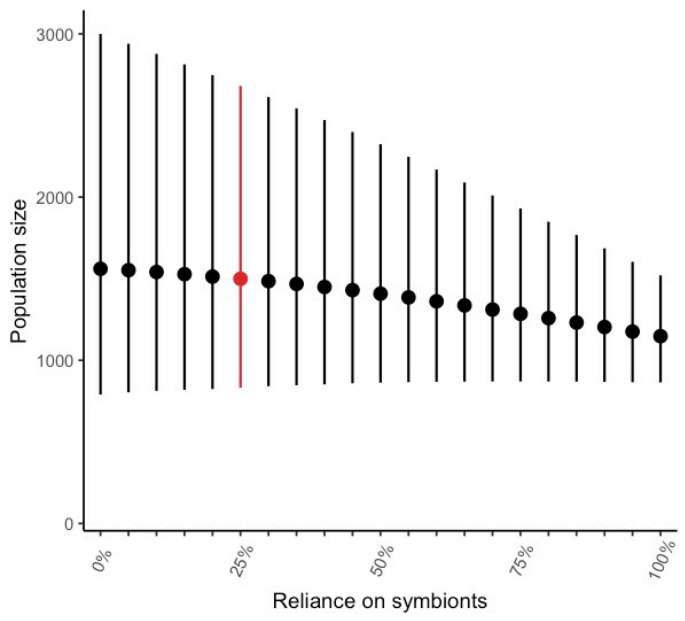

B

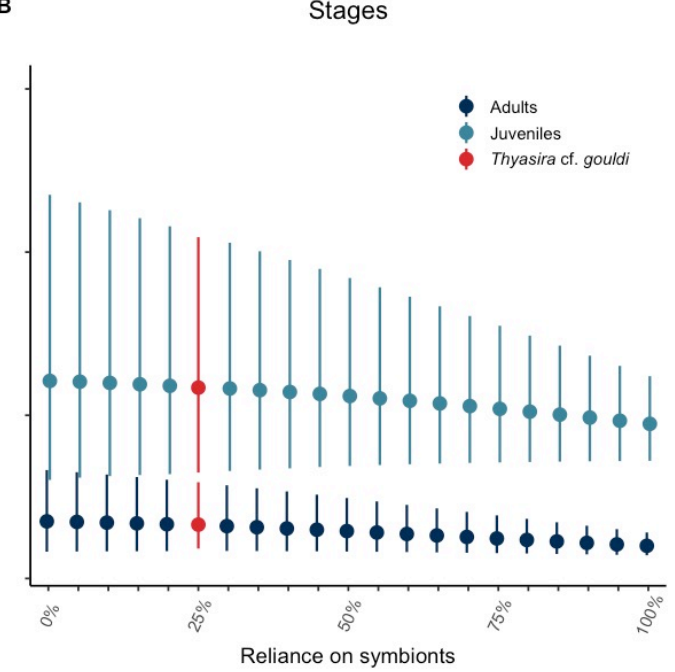

Figure 4. Effect of varying symbiont reliance $(\alpha)$ on the host populations in a seasonal environment. For all simulations, the initial population consists of five embryos. T. cf. gouldi, which has a dependence on symbionts assumed to be $25 \%$, is highlighted for reference. Points represent the average population size after discarding transient dynamics, and lines correspond to the amplitude of the yearly cycle. An increasing symbiont dependence decreases the magnitude of the annual population cycles (A). As dependence on symbionts increases, the average and maximum population sizes decrease; however, the minimum population size increases. The stage dynamics exhibit the same pattern as the total population, with juveniles dominating both species' populations (B). 
ment (Fig. 2A). A similar pattern has been found in T. sarsi, which has an intermediate reliance on bacterial symbionts (between 26 to $76 \%$ ) and exhibits a faster population growth rate, reaching larger population sizes when compared to the sympatric and less symbiont dependent T. equalis (which has a reliance below 26\%) (Dando and Spiro, 1993). However, unlike T. equalis, the asymbiotic Parathyasira have higher somatic growth rates and reach larger sizes at maturity, relative to the symbiotic T. cf. gouldi (Mariño et al., 2019). Further, our results show that both populations are composed mostly of juvenile individuals (Fig. 2B, E). A comparable population structure has been observed in Thyasira gouldi, which exhibits a bimodal distribution year-round (Blacknell, 1973). Our findings for a seasonal environment show that both populations experience yearly cycles. Similarly, for Thyasira gouldi, T. sarsi, and T. equalis empirical data has suggested that they have variable population sizes (Blacknell, 1973; Dando and Southward, 1986). Thus, despite the limited empirical evidence from other thyasirid bivalves or symbiotic animals, our results broadly agree with the literature.

\subsection{Effect of symbiosis on the host population}

In our simulations, the symbiotic population of $T$. cf. gouldi experiences oscillations of a similar amplitude relative to the asymbiotic population (Fig. 2D). When we consider a symbiotic population in which the symbionts are more abundant, the amplitude of the population cycles increases (Fig. 3). Conversely, when the individuals have a higher dependency on the symbionts, the yearly cycles have smaller amplitudes (Fig. 4). Broadly, our results indicate that the magnitude of these population cycles is likely an effect of the mixotrophic diet and reduced energy reserves of the individual hosts (Mariño et al., 2019). In general, when the free-living bacteria become a limiting resource, the symbionts provide a stable alternative nutritional source for the host (Gori et al., 2012; Viladrich et al., 2017; Rossi et al., 2017), which does not need to rely on an energy reserve. As a consequence, when the resource is abundant, hosts do not need to build up a large energy reserve. Instead, the individual hosts can allocate more energy to reproduction. This effect is evident in the constant environment when the resource is not limiting, where symbiotic thyasirids reach higher abundances than the asymbiotic population (Fig. 2A).

Benefits of symbiosis at low resource concentrations have been documented before, theoretically and experimentally, in photomixotrophic and 
aposymbiotic organisms (e.g. in ciliates and in hydra Goetsch. 1924; Muscatine and Lenhoff, 1965; Karakashian, 1963; Stabell et al., 2002). The literature agrees that, at low resource concentrations, mixotrophic populations have significantly higher growth rates than aposymbiotic populations, which is sufficient to prevent extinction. At high resource availability, theory suggests that the benefits of symbiosis are in reducing loss rates (Stabell et al., 2002), which agrees with our previous finding of lower somatic maintenance costs for the symbiotic T. cf. gouldi when compared to the asymbiotic Parathyasira (Mariño et al., 2019). The role of symbionts in building-up and performing the primary energy and carbon storage for a host has only recently been described (in the chemosymbiotic flatworm Paracatenula; Jäckle et al., 2019). Therefore, evidence agrees with our analysis and suggests a potential role of symbiosis in mitigating environmental fluctuations in populations of mixotrophic individuals.

\subsection{Model assumptions and limitations}

Our results are related to our assumptions regarding the fecundity, mortality, and competition of the individuals in the populations. For the thyasirids of Bonne Bay, we do not have enough evidence to suggest that reproduction occurs in discrete events or that there is competition for resources between the species. Therefore, in our model, reproduction occurs continuously, and each species can graze independently of the other on the free-living bacterial resource. Likewise, for simplicity and lack of detailed information, we assumed that mortality was equal for both species in all the stages. The consequences of our assumptions could influence the population structure in our results. For example, in a population of T. gouldi, it has been proposed that juveniles do not uniformly predominate because early juveniles are likely to suffer a greater mortality rate, compared to late juveniles and adults (Blacknell, 1973). Nonetheless, these simplifying assumptions are unlikely to affect the overarching pattern regarding the effect of the symbiotic strategy.

The yearly cycles experienced by both populations are a direct result of the fluctuations in temperature and its effect on the resource. We modelled the temperature oscillations according to the natural variation pattern observed in Bonne Bay. The free-living bacteria are also known to be subject to seasonal variations (Laurich et al., 2015); therefore, we used a framework derived from the Metabolic Theory of Ecology to couple the environmental temperatures to the resource abundance. Even though the abundance 
and digestion of the bacterial symbionts in T. cf. gouldi also show a cyclical trend (Laurich et al., 2015), in our formulation, we treat the symbiont abundance as constant, considering that they provide a more stable source of nutrition. Despite our assumptions, the strength of our physiologically structured model is illustrated by the differences that we revealed between the two species' populations.

\subsection{Reliance on symbionts}

Differences in individual energy allocation have suggested that for $T$. cf. gouldi the symbionts may buffer resource fluctuations (Mariño et al., 2019). Our simulations suggest that symbiotic and asymbiotic thyasirids have different adaptations to persist during winter conditions when the temperatures are low, and free-living bacteria are rare. Asymbiotic thyasirids have physiological adaptations that allow them to build a larger energy reserve, which can be mobilized more when the resource is scarce. Symbiotic thyasirids have adapted via their symbionts: the reliance on a constant supply of symbiotic bacteria buffers against the effects of seasonal lows in the abundance of freeliving bacteria. Thus, for the thyasirids from Bonne Bay, both the generalist mixotrophic and the specialist diet are equally successful strategies.

Our results motivate the question of why symbiotic thyasirids do not rely more on their symbionts, or equivalently, why asymbiotic thyasirids do not have a broader diet. Both alternatives could lead to individuals less sensitive to environmental fluctuations and more stable populations sizes (Fig. 4 A, B). Such questions are associated with the phenotypic traits that determine resource acquisition, which are thought to be defined by an intraspecific correlation between the individual morphology, physiology and behavior. In general, it is understood that there are costs that prevent the evolution of niche generalism, for example, phylogenetic constrains (Ackermann and Doebeli, 2004; Futuyma and Moreno, 1988). For symbiotic thyasirids, the gill size of the host imposes a limit to the space available for colonization by symbionts (Dufour, 2005). If the surface area of thyasirid gills shows a positive allometric relationship to body size, as observed in other chemosymbiotic bivalves with similarly filibranchiate gills (Duperron et al., 2016), the small body size of thyasirids may prevent them from harbouring the number of symbionts that would be necessary for a greater reliance (i.e., equivalent to the symbiont dependencies observed in larger bivalves). Another likely explanation is that the costs of maintaining symbionts may rise during the 
winter, due to an increment in the bioirrigation necessary to control the symbiont population size or to an increase in the digestion of symbionts (Zanzer) et al., 2019). For asymbiotic thyasirids, there may be similar phylogenetic limitations that have prevented a change in diet and have instead promoted faster somatic growth and maturation rates. Moreover, an environment with spatial variation, such as the infaunal habitat of the thyasirids, could equally favour diet specialization (Reboud and Bell, 1997). The limited evidence available for the thyasirids hinders a more robust inference; however, it is clear that both strategies are successfully maintained in the community.

The ubiquity of symbiosis makes it a crucial factor that can determine the outcome of ecological and evolutionary processes (Moran, 2006). Nevertheless, how symbiosis affects ecology and evolution remains mostly unknown. In this study, we show how trophic symbiosis can mitigate the effect of a seasonal environment in a population of bivalve hosts. Although we parameterized our model for the particular system of thyasirid bivalves from the fjord of Bonne Bay, Canada, our approach has a general nature, and our results are relevant in a variety of trophic symbiosis. Our results highlight the relevance of linking individual energetics and life history to population dynamics and are the first step towards a general understanding of the role of symbiosis in populations' resilience.

\section{Acknowledgements}

We thank the members of the Theoretical Biology Laboratory at MUN for their constructive comments on the manuscript. JM was supported by the School of Graduate Studies Baseline Fellowship from Memorial University. SCD and AH received funding from the Natural Science and Engineering Research Council of Canada (NSERC Discovery Grants 2015-06548 and 201405413

\section{Author contribution statement}

All authors conceived the ideas for the project. JM and AH designed the methodology; JM analysed the results and led the writing of the manuscript. All authors contributed critically to the drafts and gave final approval for publication. 


\section{Appendix}

\subsection{Individual model}

\subsubsection{Metabolic acceleration: DEB-abj model}

In the DEB theory framework, species with larval development typically exhibit a slow embryonic development combined with a faster development during the late juvenile and adult stages (Kooijman, 2014). This permanent increase in the metabolic rate is called metabolic acceleration. The most common type of acceleration, called $\mathcal{M}$ acceleration, involves a simultaneous increase in the assimilation and reserve mobilization rates between birth and metamorphosis, as well as an increase in growth, maturation, reproduction and respiration. We quantified the metabolic increase in type $\mathcal{M}$ acceleration according to the shape correction function:

$$
\mathcal{M}=\max \left(L_{b}, \min \left(L, L_{j}\right)\right) / L_{b},
$$

where $L$ is the length in structure, and $L_{b}$ and $L_{j}$ represent the structural lengths at the beginning and at the end of the acceleration, which correspond to the structural lengths at birth and at metamorphosis.

\subsubsection{Observables: fecundity}

The state variables of the DEB model are quantities that are not directly measurable. Hence, to calculate how the cumulative energy invested into reproduction $\left(E_{R}\right)$ translates to number of embryos per time, we used the following relation (Eq. 2.56 in Kooijman, 2010):

$$
\dot{R}=\frac{\kappa_{R} E_{R}}{E_{0}},
$$

where $\kappa_{R}$ is the reproduction efficiency (set to the standard value of 0.95 ) and $E_{0}(\mathrm{~J})$ is the amount of initial energy reserve invested into each embryo (i.e. the cost of an egg). To calculate $E_{0}$ (Eq. 2.42 in Kooijman, 2010) we used the routine initial_scaled_reserve in the DEBtool MATLAB package (Lika et al., 2014).

\subsection{Grid resolution and computational time}

We ran the models for Section 3.1 using a grid resolution of $30^{3}$ cells for ten years, measuring each day's population density. In Section 3.2, we used a grid resolution of $20^{3}$ cells for six years, with output also given every day. 
Similarly, for Section 3.3, we integrated the model for six years with output each day, but using a grid of $15^{3}$ cells.

We used an Intel Xeon E5-1650 v2 @ 3.50GHz processor to run the models. We measured the integration time in core-years (calculated as hours - (nodes · cores $) /(365 \cdot 24))$. The integration time for the models for Thyasira cf. gouldi and Parathyasira sp. in a constant environment at a resolution of $30^{3}$ cells was 0.002 and 0.012 core-years respectively. For the same grid size in the seasonal scenarios, the running time was 0.218 core-years for T. cf. gouldi and 0.232 core-years for Parathyasira. The simulations for $T$. cf. gouldi at a $20^{3}$ resolution took 0.018 core-years to complete, and 0.004 core-years using a grid size of $15^{3}$ cells.

\section{References}

Ackermann, M., Doebeli, M., 2004. Evolution of niche width and adaptive diversification. Evolution 58 (12), 2599-2612.

Bell, G., Reboud, X., 1997. Experimental evolution in Chlamydomonas II. genetic variation in strongly contrasted environments. Heredity 78 (5), 498-506.

Blacknell, W. M., 1973. Aspects of the biology of Thyasira gouldi (Philippi) and its copepod parasite Axinophilus thyasirae (Bresciani and Ockelmann). Ph.D. Thesis, University of Stirling.

Dando, P. R., Southward, A. J., 1986. Chemoautotrophy in bivalve molluscs of the genus Thyasira. Journal of the Marine Biological Association of the UK 66, 915-929.

Dando, P. R., Spiro, B., 1993. Varying nutritional dependence of the thyasirid bivalves Thyasira sarsi and T. equalis on chemoautotrophic symbiotic bacteria, demonstrated by isotope ratios of tissue carbon and shell carbonate. Marine Ecology Progress Series 92 (1-2), 151-158.

De Roos, A. M., Galic, N., Heesterbeek, H., 2009. How resource competition shapes individual life history for nonplastic growth: ungulates in seasonal food environments. Ecology 90 (4), 945-960.

De Roos, A. M., Persson, L., 2001. Physiologically structured models-from versatile technique to ecological theory. Oikos 94 (1), 51-71. 
Dubilier, N., Bergin, C., Lott, C., 2008. Symbiotic diversity in marine animals: the art of harnessing chemosynthesis. Nature Reviews. Microbiology 6 (10), 725-740. URL http://www.ncbi.nlm.nih.gov/pubmed/18794911

Dufour, S. C., 2005. Gill anatomy and the evolution of symbiosis in the bivalve family Thyasiridae. Biological Bulletin 208 (3), 200-212.

Dufour, S. C., Felbeck, H., 2006. Symbiont abundance in thyasirids (Bivalvia) is related to particulate food and sulphide availability. Marine Ecology Progress Series 320, 185-194.

Duperron, S., Gaudron, S. M., Rodrigues, C. F., Cunha, M. R., Decker, C., Olu, K., 2013. An overview of chemosynthetic symbioses in bivalves from the North Atlantic and Mediterranean Sea. Biogeosciences 10 (5), $3241-3267$.

Duperron, S., Quiles, A., Szafranski, K. M., Léger, N., Shillito, B., 2016. Estimating symbiont abundances and gill surface areas in specimens of the hydrothermal vent mussel Bathymodiolus puteoserpentis maintained in pressure vessels. Frontiers in Marine Science 3, 16.

Fabricius, K. E., Genin, A., Benayahu, Y., 1995. Flow-dependent herbivory and growth in zooxanthellae-free soft corals. Limnology and Oceanography 40 (7), 1290-1301.

Ferrier-Pagès, C., Reynaud, S., Béraud, E., Rottier, C., Menu, D., Duong, G., Gévaert, F., 2015. Photophysiology and daily primary production of a temperate symbiotic gorgonian. Photosynthesis Research 123 (1), 95-104.

Futuyma, D. J., Moreno, G., 1988. The evolution of ecological specialization. Annual Review of Ecology and Systematics 19 (1), 207-233.

George, S. B., 1994. Population differences in maternal size and offspring quality for Leptasterias epichlora (brandt) (echinodermata: Asteroidea). Journal of Experimental Marine Biology and Ecology 175 (1), 121-131.

Goetsch, W., 1924. Die symbiose der süsswasser-hydroiden und ihre künstliche beeinflussung. Zeitschrift für Morphologie und Ökologie der Tiere 1 (4), 660-751. 
Gori, A., Viladrich, N., Gili, J. M., Kotta, M., Cucio, C., Magni, L., Bramanti, L., Rossi, S., 2012. Reproductive cycle and trophic ecology in deep versus shallow populations of the Mediterranean gorgonian Eunicella singularis (Cap de Creus, northwestern Mediterranean Sea). Coral Reefs 31 (3), 823-837.

Grottoli, A. G., Rodrigues, L. J., Palardy, J. E., 2006. Heterotrophic plasticity and resilience in bleached corals. Nature 440 (7088), 1186-1189.

Jäckle, O., Seah, B. K., Tietjen, M., Leisch, N., Liebeke, M., Kleiner, M., Berg, J. S., Gruber-Vodicka, H. R., 2019. Chemosynthetic symbiont with a drastically reduced genome serves as primary energy storage in the marine flatworm Paracatenula. Proceedings of the National Academy of Sciences 116 (17), 8505-8514.

Karakashian, S. J., 1963. Growth of Paramecium bursaria as influenced by the presence of algal symbionts. Physiological Zoology 36 (1), 52-68.

Kassen, R., 2002. The experimental evolution of specialists, generalists, and the maintenance of diversity. Journal of Evolutionary Biology 15 (2), 173190.

Kassen, R., Bell, G., 1998. Experimental evolution in Chlamydomonas. IV. selection in environments that vary through time at different scales. Heredity 80 (6), 732-741.

Kooijman, S. A. L. M., 2010. Dynamic Energy Budget theory for metabolic organisation, 3rd Edition. Cambridge University Press, Cambridge.

URL http://www.pubmedcentral.nih.gov/articlerender.fcgi? artid $=2981979\{\&\}$ tool=pmcentrez $\{\&\}$ rendertype=abstract

Kooijman, S. A. L. M., 2014. Metabolic acceleration in animal ontogeny: An evolutionary perspective. Journal of Sea Research 94, 128-137.

URL http://dx.doi.org/10.1016/j.seares.2014.06.005

Laurich, J. R., Batstone, R. T., Dufour, S. C., 2015. Temporal variation in chemoautotrophic symbiont abundance in the thyasirid bivalve Thyasira cf. gouldi. Marine Biology 162 (10), 2017-2028.

Levins, R., 1968. Evolution in changing environments: some theoretical explorations. Princeton University Press. 
Lika, K., Augustine, S., Pecquerie, L., Kooijman, S. A., 2014. The bijection from data to parameter space with the standard DEB model quantifies the supply-demand spectrum. Journal of theoretical biology 354, 35-47.

Lynch, M., Gabriel, W., 1987. Environmental tolerance. The American Naturalist 129 (2), 283-303.

Mariño, J., Augustine, S., Dufour, S. C., Hurford, A., 2019. Dynamic Energy Budget theory predicts smaller energy reserves in thyasirid bivalves that harbour symbionts. Journal of Sea Research 143, 119-127.

Martin, B. T., Jager, T., Nisbet, R. M., Preuss, T. G., Grimm, V., 2013. Predicting population dynamics from the properties of individuals: A crosslevel test of Dynamic Energy Budget theory. The American Naturalist 181 (4), 506-519. URL http://www.jstor.org/stable/10.1086/669904

Martin, B. T., Zimmer, E. I., Grimm, V., Jager, T., 2012. Dynamic Energy Budget theory meets individual-based modelling: A generic and accessible implementation. Methods in Ecology and Evolution 3 (2), 445-449.

McKillup, S. C., Butler, A. J., 1979. Modification of egg production and packaging in response to food availability by Nassarius pauperatus. Oecologia 43 (2), 221-231.

Miller, T. E., Rudgers, J. A., 2014. Niche differentiation in the dynamics of host-symbiont interactions: symbiont prevalence as a coexistence problem. The American Naturalist 183 (4), 506-518.

Moran, N. A., 2006. Symbiosis. Current Biology 16 (20), 866-871.

Muller-Parker, G., Davy, S. K., 2001. Temperate and tropical algal-sea anemone symbioses. Invertebrate Biology 120 (2), 104-123.

Muscatine, L., Lenhoff, H. M., 1965. Symbiosis of hydra and algae. II. effects of limited food and starvation on growth of symbiotic and aposymbiotic hydra. The Biological Bulletin 129 (2), 316-328.

Nisbet, R. M., Jusup, M., Klanjscek, T., Pecquerie, L., 2012. Integrating dynamic energy budget (DEB) theory with traditional bioenergetic models. The Journal of Experimental Biology 215 (6), 892-902. 
URL http://jeb.biologists.org/cgi/doi/10.1242/jeb. 059675\{\%\}5Cnhttp://www.ncbi.nlm.nih.gov/pubmed/22357583

Pile, A. J., Young, C. M., 1999. Plankton availability and retention efficiencies of cold-seep symbiotic mussels. Limnology and Oceanography 44 (7), $1833-1839$.

R Core Team, 2019. R: A Language and Environment for Statistical Computing. R Foundation for Statistical Computing. URL https://www.R-project.org/

Reboud, X., Bell, G., 1997. Experimental evolution in Chlamydomonas. III. evolution of specialist and generalist types in environments that vary in space and time. Heredity 78 (5), 507-514.

Rossi, S., Coppari, M., Viladrich, N., 2017. Benthic-pelagic coupling: new perspectives in the animal forests. In: Rossi, S., Bramanti, L., Gori, A., Orejas, C. (Eds.), Marine animal forests: the ecology of benthic biodiversity hotspots. Springer International Publishing, pp. 855-885.

Sander, B. C., Kalff, J., 1993. Factors controlling bacterial production in marine and freshwater sediments. Microbial Ecology 26 (2), 79-99.

Savage, V. M., Gillooly, J. F., Brown, J. H., West, G. B., Charnov, E. L., 2004. Effects of body size and temperature on population growth. The American Naturalist 163 (3), 429-441.

Schoolfield, R., Sharpe, P., Magnuson, C., 1981. Non-linear regression of biological temperature-dependent rate models based on absolute reactionrate theory. Journal of Theoretical Biology 88 (4), 719-731.

Soetaert, K., Petzoldt, T., Setzer, R. W., 2010. Solving differential equations in R: Package deSolve. Journal of Statistical Software 33 (9), 1-25. URL http://www.jstatsoft.org/v33/i09

Sorokin, Y., 1991. Biomass, metabolic rates and feeding of some common reef zoantharians and octocorals. Marine and Freshwater Research 42 (6), 729-741.

Southward, E. C., 1986. Gill symbionts in thyasirids and other bivalve molluscs. Journal of the Marine Biological Association of the United Kingdom $66,889-914$. 
Stabell, T., Andersen, T., Klaveness, D., 2002. Ecological significance of endosymbionts in a mixotrophic ciliate - an experimental test of a simple model of growth coordination between host and symbiont. Journal of Plankton Research 24 (9), 889-899.

Taylor, J. D., Williams, S. T., Glover, E. A., 2007. Evolutionary relationships of the bivalve family Thyasiridae (Mollusca: Bivalvia), monophyly and superfamily status. Journal of the Marine Biological Association of the United Kingdom 87 (02), 565-574.

URL http://dx.doi.org/10.1017/S0025315407054409

Thompson, R., 1983. The relationship between food ration and reproductive effort in the green sea urchin, Strongylocentrotus droebachiensis. Oecologia $56(1), 50-57$.

van der Meer, J., 2006. Metabolic theories in ecology. Trends in Ecology and Evolution 21 (3), 136-140.

Viladrich, N., Bramanti, L., Tsounis, G., Martínez-Quintana, A., FerrierPagès, C., Rossi, S., 2017. Variation of lipid and free fatty acid contents during larval release in two temperate octocorals according to their trophic strategy. Marine Ecology Progress Series 573, 117-128.

Yule, K. M., Miller, T. E., Rudgers, J. A., 2013. Costs, benefits, and loss of vertically transmitted symbionts affect host population dynamics. Oikos 122 (10), 1512-1520.

Zanzerl, H., Salvo, F., Jones, S. W., Dufour, S. C., 2019. Feeding strategies in symbiotic and asymbiotic thyasirid bivalves. Journal of sea research 145, $16-23$. 